\title{
Research on the test scheme of power time synchronization test network
}

\author{
Xiong Hongzhang ${ }^{1, a}$, Gao Qiang ${ }^{1, b}$, Teng Ling ${ }^{1, \mathrm{c}}$, He Chen $^{2, \mathrm{~d}}$, Wang Yang ${ }^{1, \mathrm{e}}$, \\ Chen $\mathrm{Xi}^{1, \mathrm{f}}$
}

${ }^{1}$ China Electric Power Research Institute, Beijing, 100192, China

${ }^{2}$ State Grid Zhejiang Information \& Telecommunication Branch Company, Hangzhou, 310006 , China

aemail: xhongzhang@foxmail.com, bemail: gaoqiang2010@epri.sgcc.com.cn

cemail: tengling@epri.sgcc.com.cn, ${ }^{\mathrm{d} e m a i l}$ :herohc@126.com

eemail: yangw@epri.sgcc.com.cn, ${ }^{\mathrm{f}}$ email: chenxi@epri.sgcc.com.cn

\section{Keywords: Smart Grid; Power Time Synchronization; Test Network; Test Scheme}

\begin{abstract}
With the development of the smart grid construction, the size of the grid is growing, the business types and the demand are gradually increasing and the high standards of the time synchronization performance transmission put forward higher requirements. This article introduces the power time synchronization network situation and the demand of time synchronization status and analysis of the power time synchronization methods, analysis the time synchronization experiment network in PTP protocol through SDH E1 line of transmission principle, according to the effect of the application of the time synchronization experiment network, gives the Suggestions to the construction of time synchronized.
\end{abstract}

\section{Introduction}

As the grid has become increasingly complex and intelligent degree unceasing enhancement, in order to make sure the security, stable and economic operation of the grid, all kinds of equipments that based on automation technology, computer technology and communication technology are widely used. The normal operation of the device is inseparable from the unified time base ${ }^{[1]}$.

The existing power system is a simple way to get the time information through the GPS/ Beidou satellite receiver in the internal configuration of the substation. Due to the decentralized management of the station that did not form a unified network time. Each power plant and substation and dispatching center is islands of time. The time system of the engine room there is no unified precision requirements and safety standards ${ }^{[2]}$.

In recent years, with the advance of the construction of smart grid, the power system has become more and more important to the time synchronization. The unified planning and construction of time synchronization network have become an urgent problem to be solved.

\section{Overview of power time synchronization network}

The power system's station substation and power plant at all levels are equipped with a time synchronization system. Time synchronization system has a variety of ways, which includes the typical form includes basic, master-slave and master-standby. The basic time synchronization system consists of a master clock and the signal transmission medium, for timing device or system. Master-slave synchronization system includes a master clock, several slave clocks and the signal transmission medium composition, for timing device or system. At present, the State Grid Corporation mainly use master-standby synchronization system, in order to improve the reliability of the time synchronization system, master-standby time synchronization system is composed by two main clocks, several slave clocks and the signal transmission medium, for timing device or system. According to the actual needs and technical requirements, the three composition time 
synchronization system of the master clock can be left with an interface, to receive the superior time synchronization system of cable time reference signal.

The China State Grid Corp at this stage has not yet completed the unified time synchronization network, only a handful of companies to carry out the construction and use of time synchronization network test, most stations provide synchronization system for station equipment through the respective time signal, and the satellite is a single time reference source, there are many shortcomings, mainly reflected in the following aspect: the GPS satellite system is the only clock source exists political security risks; satellite system as the only time reference makes the low reliability of synchronous, independent operation of the synchronization system, the lack of clock devices in the area to centralized monitoring and control of the means and methods of regional plant.

\section{Demand status of power time synchronization}

Equipment or system in power system that needs time synchronization include different dispatching institutions, protection or automation equipment that in power plant and substation, communication network management system, computer system and based on computer information systems (such as toll system, management information system), image monitoring system, electronic clock and power system.

The timing device of Power system that in accordance with the business can be classified into three categories. The first class is used to protect the equipment, mainly used for transformer protection, lines, circuit breakers and other important device. The second is an automation equipment, mainly used for improve the equipment running state monitoring, measurement, control and coordination level. The third category includes communication network management system, computer system, electronic clock and other equipment. Protection and automation equipment for time accuracy requirements can be as high as microsecond, substation of precision requirements that higher than 1 microsecond commonly used by timing device has three kinds: traveling wave fault location device, a synchronous phasor measuring device and lightning location system. Communication network management system and computer system for the time accuracy of the highest level are only milliseconds.

\section{Power time synchronization mode}

From the power time synchronization development process, power time synchronization methods mainly include 1PPS+ToD, B time code, NTP and PTP time synchronization mode. Power time synchronization system early mainly uses 1 PPS + ToD to realize time synchronization. Using the pulse on time delay (rising edge or falling edge) to calibrate the timing equipment. Because the 1PPS+ToD manner needs to pass the 2 signal and data needs to be manually set, hence the IRIG-B code time way. B code can be sent via the serial communication interface to timing equipment, $\mathrm{B}$ time code synchronization precision is high, up to 1 microsecond, which can meet the demand of time synchronization for most of the electric power business, and most of the device of power system have adopted the B code synchronization mode now. In the meantime, NTP based time synchronization in power system has also been applied, which can realize the synchronization accuracy of LAN millisecond. At the beginning of 2009, with a small amount of equipment using PTP manner to realize the functions of time synchronization, and the synchronization precision can reach 1 microsecond ${ }^{[3]}$.

IEEE1588 defines a protocol for achieving high precision clock synchronization in the measurement and control system. PTP protocol integrates many technologies, such as network communication, local computation and distributed object, which can be used in the distributed system of communication ${ }^{[4]}$. Power time synchronization test network mainly uses PTP protocol to transmit time synchronization signal to meet the requirements of power system for time synchronization. 


\section{PTP over SDH transmission principle}

$\mathrm{SDH}$ is the main communication network of power and SDH $2 \mathrm{M}$ resource is rich. Through SDH $2 \mathrm{M}$ to transmit PTP that build time synchronization network, which does not require any modification on the existing SDH network and PTP as a communication service in SDH 2M that achieve transparent transmission ${ }^{[5]}$.

At present, the SDH device itself can't support PTP, and the time synchronization signal must be converted to the E1/Ethernet protocol through the transmission. The PTP packet protocol is transmitted through the SDH E1 line, and the three order high density bipolar code (HDB3) stream and the Ethernet protocol stream are converted to E1.

The principle of PTP protocol through E1 line is: master clock through Ethernet to send protocol message. After E1/Ethernet protocol converter, which turn Ethernet bit stream into E1 HDB3 code stream, and sent through a SDH network step by step, in the reach from the slave clock, through the association of E1/Ethernet protocol converter E1 HDB3 code stream into Ethernet stream, and transfer to the slave clock ${ }^{[6]}$. The message is transmitted to the master clock by a similar process from the slave clock, and the slave clock is calculated and adjusted according to the hardware time stamp of the packets.

\section{Test scheme for power time synchronization test network}

In order to meet the demand of time synchronization in power business, some companies in recent years have carried out the pilot work of time synchronization network. This article is based on the electric power network time synchronization test scheme for related research.

Power time synchronization network test takes provincial power company as a clock node, the Regional Bureau as secondary clock node, substation as tertiary clock node, PTP protocol is used in the whole network, which based on SDH network construction company time synchronization network time link, realize regional electric power bureau and substation time with the provincial power company synchronization. Provincial Power Company of time synchronization network level PTP master clock built-in rubidium clock, in addition to the GPS reference source, we can also receive ground output link of E1 signal and possible future increase in Beidou clock output reference signal, so as to ensure the reliability of a PTP master clock time reference source.

Provincial Power Company configuration of the PTP switches realize PTP time signal distribution function, which can meet provincial power company a PTP master clock and Regional Bureau of secondary PTP slave clock for synchronization. Implementation of PTP over SDH by using PTP/E1 protocol converter (the PTP packet is converted to E1 format). The regional power station each station is configured with one $\mathrm{PTP} / \mathrm{E} 1$ protocol converter and one PTP slave clock. The $\mathrm{PTP} / \mathrm{E} 1$ protocol converter converts the SDH device of the E1 signal into a PTP signal, which is transmitted to the two stage PTP slave clock.

Secondary PTP slave clock includes one set of rubidium clock, a high stability crystal clock, as the master clock of the Regional Electric Power Bureau, also can be used as boundary clock output PTP signal. Substation of time synchronization network structure and regional electric power bureau is similar, the difference lies in the substation of PTP slave clock can be as the main clock, transmission of IRIG-B signal to timing the station existing time synchronization system, so as to realize the time synchronization of station of the time synchronization system with the provincial power companies.

Time synchronization network topology as shown in Figure 1, the time synchronization analyzer measured substation in three levels of PTP master clock which includes light Interface B code, electric Interface B code and clock expansion unit time precision and stability of output difference B code. Test results are shown in Figure 2, Figure 3 and Figure 4. 


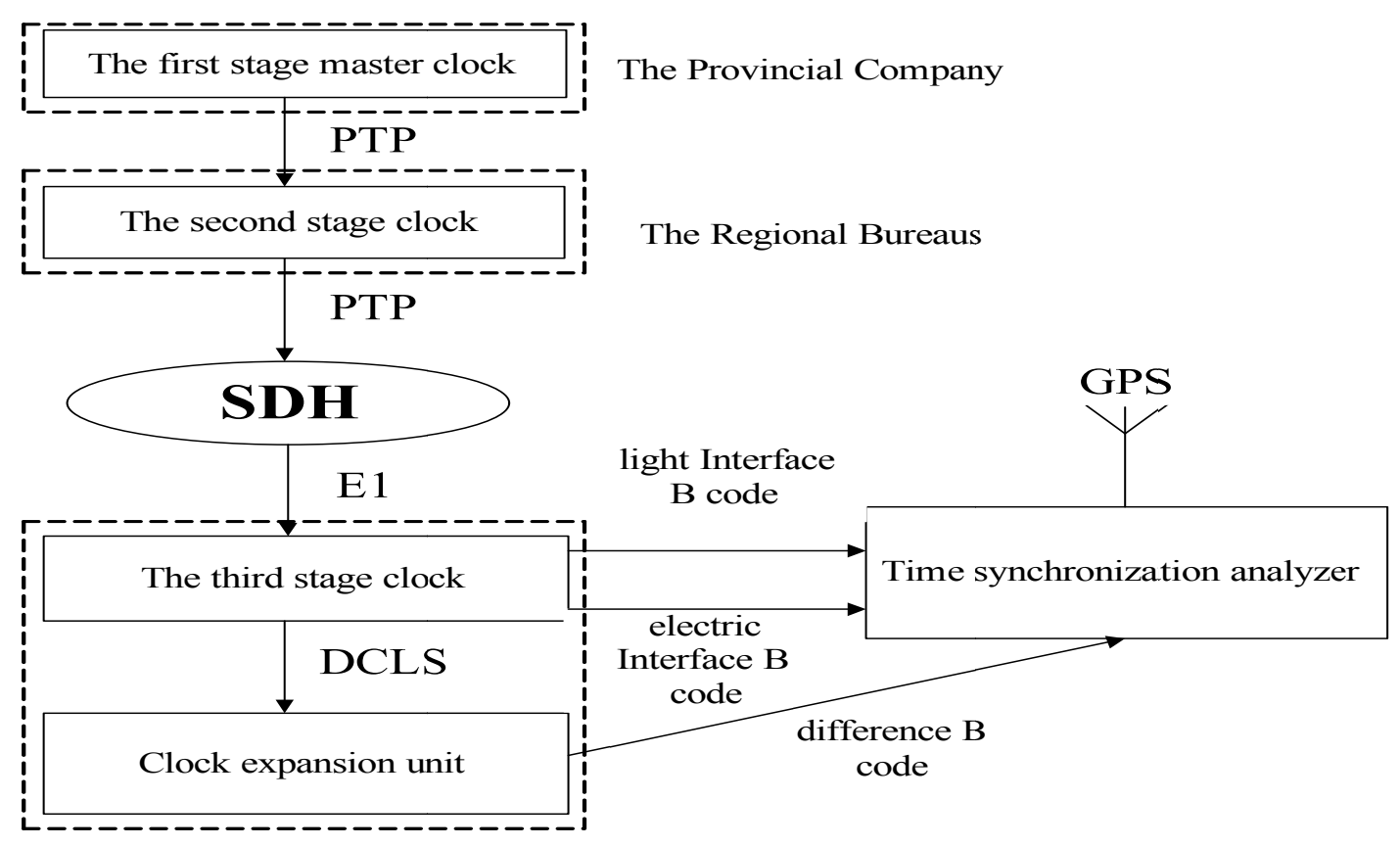

The Substations

Figure 1: Topological graph of time synchronization network

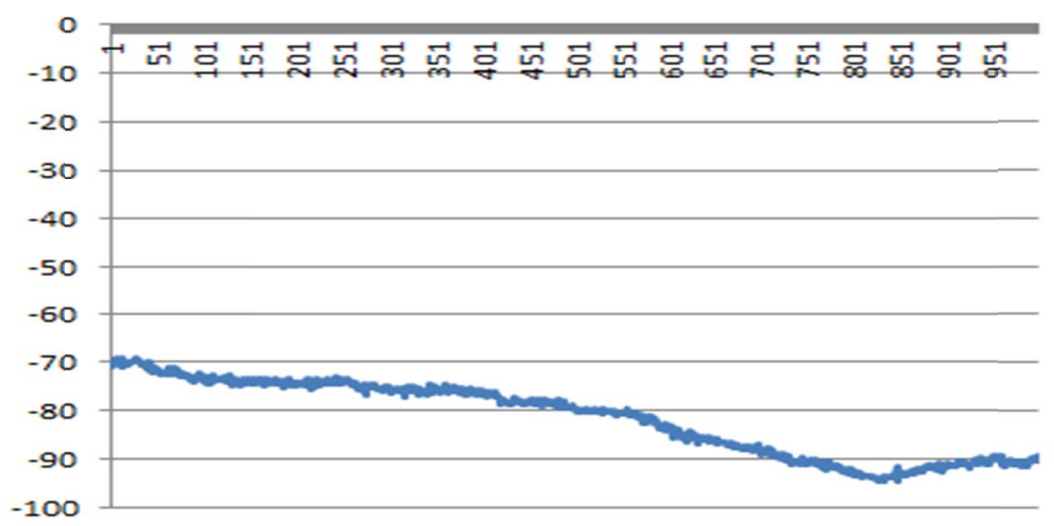

Figure 2: Test results of light interface B code

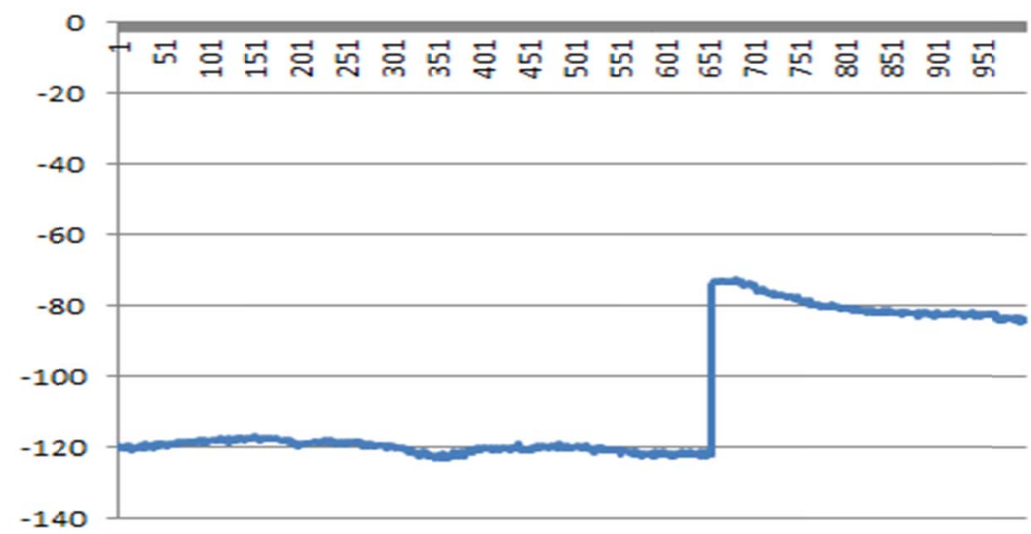

Figure 3: Test results of electric interface B code 


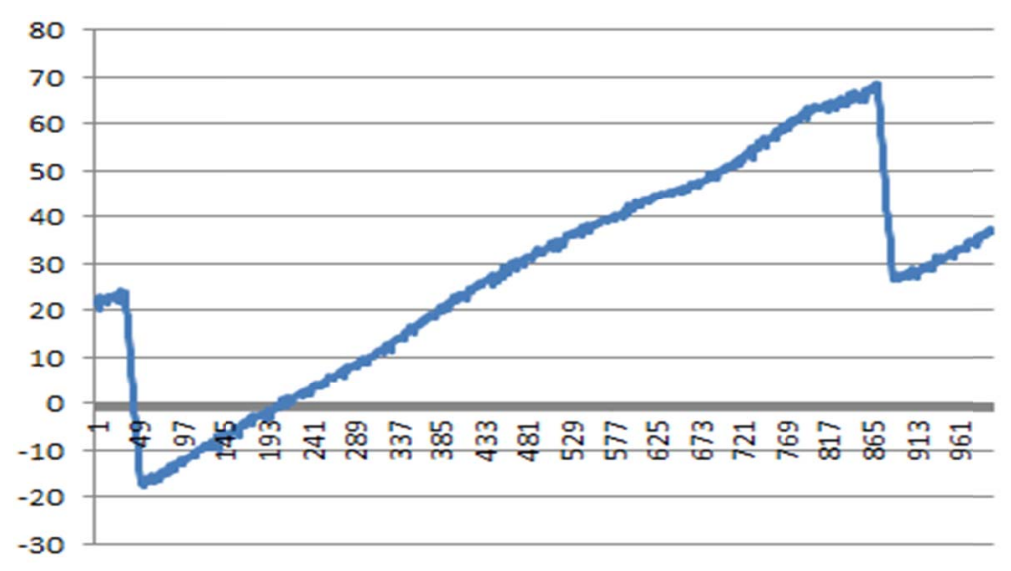

Figure 4: Test results of difference interface B code

\section{Conclusion}

By comparing substation's PTP master clock of light interface B code, electric interface B code and difference interface $\mathrm{B}$ code of synchronization interface performance, from the test of time performance of the maximum value, minimum value, average value and changes in peak indicators, we can see that light interface B code synchronization performance is better. By comparing PTP master clock and clock expansion unit output time synchronization performance, from the test of time performance of the maximum value, minimum value, average value, we can see that clock expansion unit output time synchronization performance is better. By comparing the changes of peak, PTP master clock output time synchronization can be better. Overall, the power time synchronization test network synchronization performance index is good, which can meet the accuracy requirements of 1 microsecond.

\section{Acknowledgement}

In this paper, the research was sponsored by the Science and technology project of State Grid Corporation of China (SGCC) (Project No.XX71-14-042)

\section{References}

[1] Yu yuehai, Zhangdaonong, Huyonghui. Power system time synchronization scheme[J]. Automation of Electric Power Systems. 2008, 32(4):82-86.

[2] Zhang lan, Zhangbin. Discussion on the construction of time synchronization network in electric power system[J].Telecommunications for Electric Power System. 2007, 28(171):23-27.

[3] Chen yezhao. Research on the technology of power time synchronization network[J]. GUANGDONG ELECTRIC POWER.2007,20(06):41-44.

[4] Li xiaozhen, Sujianfeng. Study on high accuracy network Clock synchronization based on IEEE 1588[J].Communications Technology.2011, 44(03):105-110.

[5] Li jian.PTP OVER SDH Power Grid Timing Synchronization Network Application [J].JIANGXI ELECTRIC POWER.2011, 35(05):12-14.

[6] Zhang jirong, Fangqiang, Xudongming. Delay Analysis and Emulation on SDH Network Elements[J]. ACTA PHOTONICA SINICA.2008,37(05):875-878. 